\title{
Übungsunterricht Strahlentherapie - fit für die Zukunft?
}

\section{Einleitung}

Vor vier Jahren haben wir uns an dieser Stelle Gedanken über die zukünftige Ausbildung im Bereich Strahlentherapie gemacht. Die MTRA-Schule in Nürnberg verfügt seit ihrer Gründung im Jahr 1991 über ein schuleigenes Übungsgerät (Therapiesimulator), an dem kein Patientenbetrieb läuft und somit ein uneingeschränkter Zugriff besteht. Dies ist ein nicht zu unterschätzender Vorteil, da eine Verwendung von im Patientenbetrieb laufenden Beschleunigern oder Simulatoren bei der hohen Auslastung dieser Geräte meist bedeutet, dass der Unterricht nur zu ungünstigen Zeiten stattfinden kann. Da aber Simulatoren heute kaum noch gebräuchlich sind, haben diese auch für den Schulbetrieb keine Zukunft weil die Räumlichkeiten in der Strahlentherapie für andere Geräte benötigt werden (z.B. Computertomograf für die virtuelle Simulation) oder Ersatzteile nicht mehr zu Verfügung stehen. Ein „virtueller Beschleuniger" kam für uns nicht infrage, da die Nachteile die Vorteile bei Weitem überwiegen. So fehlt beispielsweise das haptische Erleben komplett: Der Patient (Übungspuppe) wird hier praktisch durch ein Hologramm ersetzt, das nur mit der Maus bewegt wird und keine Anzeichnungen von z.B. Lasermarkierungen erlaubt.

\section{Schulgeräte im Wandel der Zeit}

Seit dem Gründungsjahr 1991 konnte die Schule auf einen alten Therapiesimulator ( Abb. 1) zurückgreifen. Das aus den 70er Jahren stammende Gerät hatte noch keine isozentrische Rotationsmöglichkeit, konnte dadurch aber in einem „normalen“ Raum betrieben werden. Mit dem Wechsel auf einen moderneren, isozentrisch drehbaren Simulator im Jahr 2008 ( $>$ Abb. 2) wurde ein kompletter Neubau notwendig, finanziert durch eine von unserem Strahlentherapeuten gegründete Stiftung. Die Gesamtkosten betrugen ca. $150000 €$ ( $\triangleright$ Abb. 3). Die langfristig schlechte Ersatzteilversorgung ließ uns vor einigen Jahren mit Überlegungen beginnen, wie so ein Gerät mittelfristig zu ersetzen wäre.

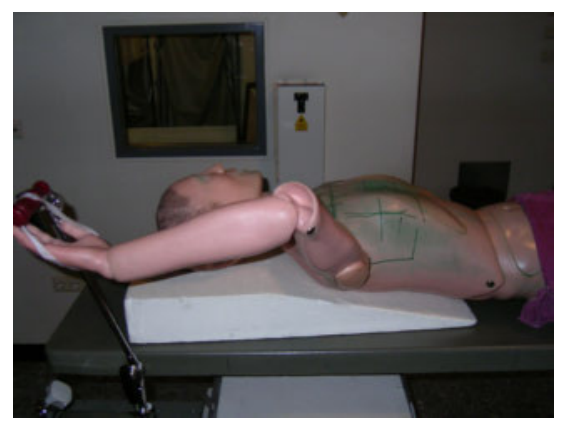

- Abb. 1 Therapiesimulator aus den 1970er Jahren.

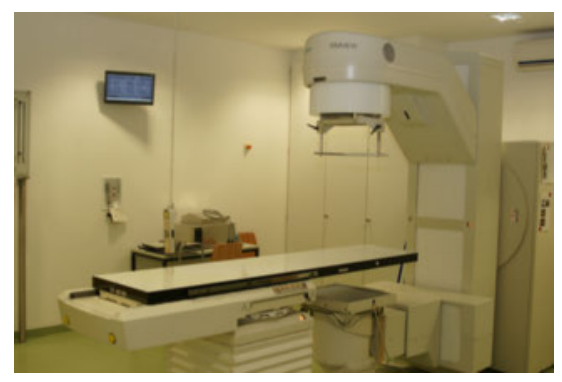

- Abb. 2 Isozentrisch drehbarer Simulator aus dem Jahr 2008.

Aus den oben genannten Gründen kam ein virtuelles System für unsere Ausbildungsstätte nicht infrage. Doch was könnte eine Alternative sein? Der Einbau eines relativ neuen gebrauchten Simulators hätte das Problem lediglich ein paar Jahre in die Zukunft verschoben, eine nachhaltige Lösung für die nächsten 15-20 Jahre wäre wünschenswert.

Eine Idee war, einen Tisch eines Simulators oder Beschleunigers ohne Gantry, lediglich mit dem Laserpositionierungssystem zu betreiben. In Kombination mit einer leeren Beschleunigerhülle (also einem Gehäuse ohne die für die hochenergetische Strahlung nötigen technischen Apparaturen) hätte so eine Maschine für die schulischen Anforderungen vielleicht schon genügt. Da jedoch noch niemand so eine Anlage jemals in Betrieb genommen hat, muss hier Überzeugungsarbeit geleistet werden - bei der technischen Entwicklung wie auch der kaufmännischen Abteilung der Gerätehersteller (Elekta bzw. Varian).

Der Einbau eines ausgemusterten, aber technisch einwandfreien und optisch

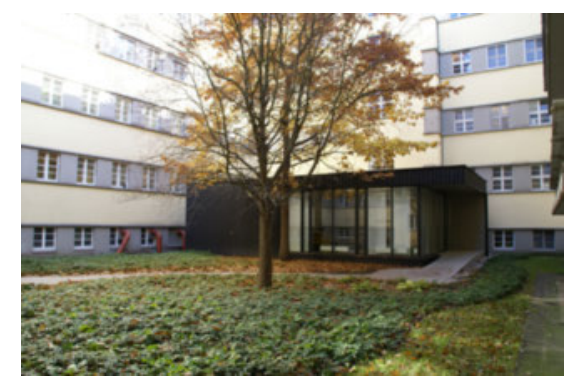

Abb. 3 Der neu gebaute Lehrkubus.

überarbeiteten („refurbished“) Linearbeschleunigers scheint hier die günstigere Variante zu sein, da es heute in größeren Serien produziert wird und - trotz aller Komplexität - mit großer Routine an jedem Ort der Welt aufgestellt werden kann. Ein gerade 10 Jahre altes, technisch intaktes Gerät, das normalerweise zur Verschrottung käme, kann so einer weiteren Verwendung zugeführt werden. Jedoch liegen die Kosten für den Umbau des Gebäudes und den Einbau des LINACs (,linear accelerator", Linerabeschleuniger) bei rund $100000 €$.

\section{Technische Herausfor-}

\section{derungen}

Der Einbau des Simulators erforderte v.a. deswegen einen Neubau, weil die Bodengrube für die isozentrische Tischrotation einen festen und tiefen Unterbau benötigt. Beim Wechsel vom SimView 3000 auf einen Elekta Precise Linearbeschleuniger ( $\triangleright$ Abb. 4) wurde ein Umbau dieser Bodengrube notwendig. Das komplette Isozentrum musste um $40 \mathrm{~cm}$ versetzt werden, um den LINAC in den vorhandenen Raum setzen zu können. Ein Linearbeschleuniger, der strahlungsfrei gemacht und in einem reinen Schulungszentrum installiert wird, war auch für den Hersteller Neuland. So musste der CEO seine Zustimmung geben, da die üblichen Prozessabläufe hier nicht einfach übernommen werden konnten. Ohne Strahlung benötigt ein LINAC keinen Anschluss an einen externen Wasserkreislauf, ohne diesen neigten anfangs einige Bauteile zur Überhitzung. Nacheinander wurden verschiedene Komponenten vom Netz genommen, um 


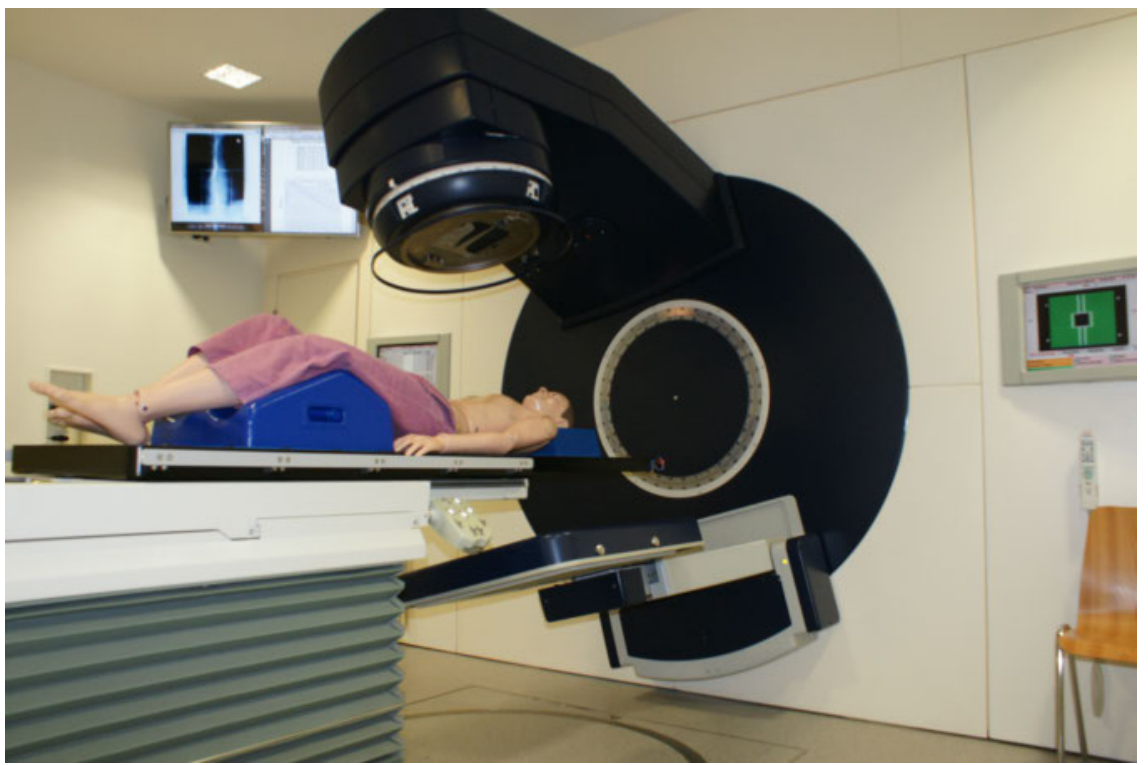

- Abb. 4 Elekta Precise Linearbeschleuniger.

die thermischen Probleme in den Griff zu bekommen. Gleichzeitig soll der Beschleuniger aber für den Schulbetrieb uneingeschränkt einsatzfähig sein, ohne dass Fehlermeldungen zu einer Störung führen. Dank der versierten Servicetechniker wurden diese Probleme sehr schnell gelöst. Für die Demontage des Altgeräts, den Umbau des Gebäudes und den Einbau des Linearbeschleunigers konnte der Zeitrahmen von 4 Monaten eingehalten werden.

Ein Vorteil dieser Lösung ist, dass für klinische Beschleuniger Ersatzteile verfügbar sind. Allerdings sind die Kosten dafür nicht unerheblich. Da für unser Gerät beim Vorbesitzer ein umfassender Wartungsvertrag bestand, wurde uns erfreulicherweise eine große Menge an Ersatzteilen quasi „gratis“ mitgeliefert, wodurch die Folgekosten reduziert werden. Die fehlende Strahlung und der vergleichsweise geringe Betrieb an der MTRA-Schule wirken sich voraussichtlich positiv auf die Lebenserwartung des Gerätes aus.

\section{Vorteile der „Hardwarelösung“}

Ein Linearbeschleuniger dieses Typs hat ein Gewicht von rund 12t. Diese Masse gefahrlos um den Patienten bzw. den Dummy rotieren zu lassen, erfordert eine gewisse Umsicht und Routine. Das Übungsgerät bietet hierfür die optimale Umgebung, da im klinischen Alltag durch Stress und Zeitdruck ein gutes Schulklima nicht so einfach entsteht.
Auch die Eingabemaske, um Bestrahlungsfelder anzulegen, kommt dem Schulalltag entgegen: Man muss Felder bezeichnen, Elektronen oder Photonen auswählen, Energien eingeben, Monitorunits, Gantryund Colliwinkel und natürlich Feldgrößen und Zubehör ( $\bullet$ Abb. 5). Fehlende oder falsche Eingaben werden vom Gerät angezeigt und können vom Lernenden korrigiert werden. Ein virtuelles System kann dies natürlich genauso gut. Aber Kollegen, die Erfahrungen sowohl mit virtuellen Systemen als auch mit physisch vorhandenen
- Abb. 5 Die Eingabemaske für Elektronen.
Übungsgeräten haben, berichten: Schüler kommen in der praktischen Ausbildung besser mit den Bestrahlungsgeräten zurecht, wenn sie an der Schule an einer hardwarebasierten Maschine geübt haben. Sicher kann man dies mit einem Fahrsimulator an einer Fahrschule vergleichen: Hier werden heutzutage einige Grundlagen gefahrlos eingeübt, bevor es in den „echten“ Straßenverkehr geht. Ein kompletter Ersatz eines Schulfahrzeugs durch eine virtuelle Realität scheint aber auf absehbare Zeit nicht sinnvoll.

Wir glauben, an unseren Schülern über die letzten Jahre eine Veränderung der Kompetenzen zu beobachten: Die nachrückenden „digital natives“ sind mit Eingabemasken und Touchscreens relativ schnell vertraut, jedoch bleiben das räumliche Denken und mechanische Fähigkeiten dahinter zurück. So bereitet das gefahrlose und rückenschonende Einsetzen eines Elektronentubus oder anderen Zubehörs meist Schwierigkeiten. Dies kann an einem virtuellen System nicht ausreichend geübt werden.

\section{Fazit}

Ein unschlagbarer Vorteil des virtuellen Beschleunigers ist das Sichtbarmachen der Strahlung und des Innenlebens des Patienten. Demgegenüber steht die fehlende Plastizität und somit auch Authentizität bzw. Realitätsnähe, welche ein physisch

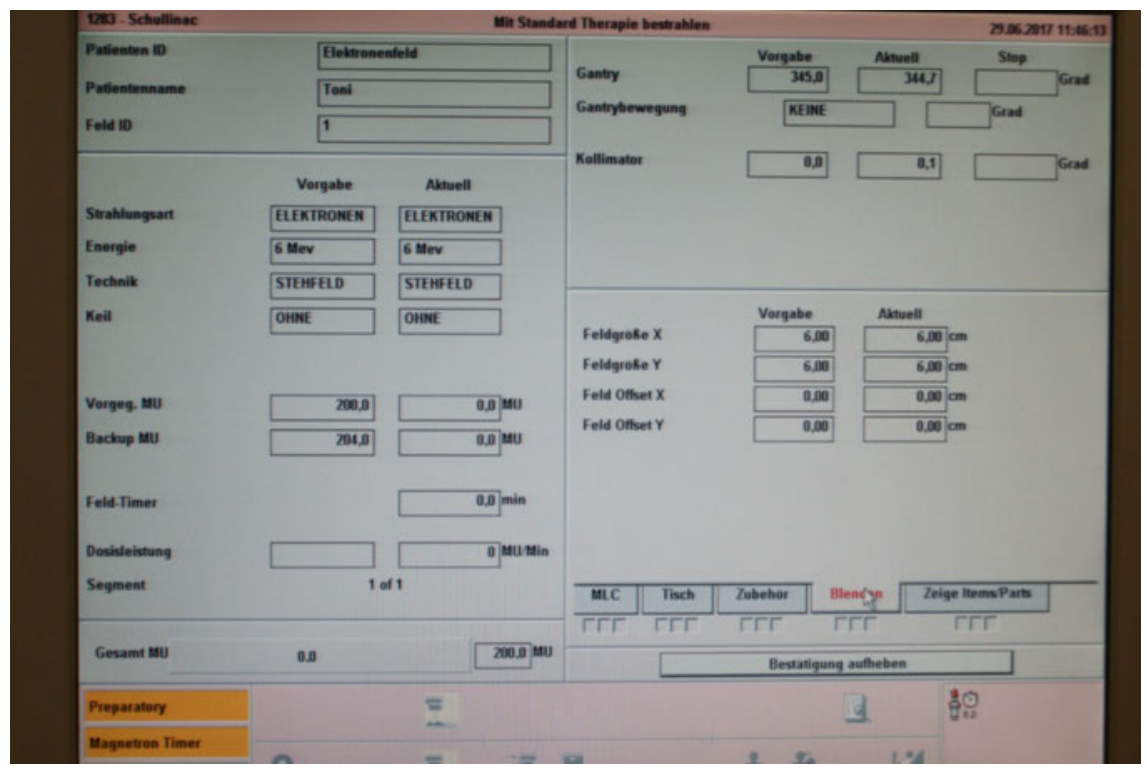


vorhandenes System aufweist ( $\triangleright$ Abb. $\mathbf{6}$,

- Abb. 7). Würde eine geregelte Aufbereitung und Zweitverwertung von Linearbeschleunigern von den Herstellern intensiver unterstützt und somit standardisiert, könnte die Hardwarelösung auch finanziell eine interessante Alternative zur virtuellen Lösung sein.

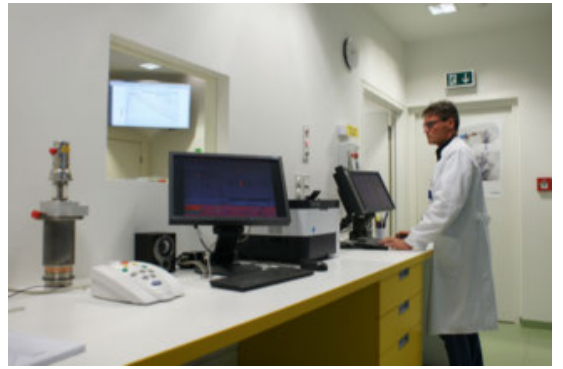

Abb. 6 Hartmut Dörwaldt bereitet den Übungsunterricht Strahlentherapie vor. Quelle: Katja Röhr.

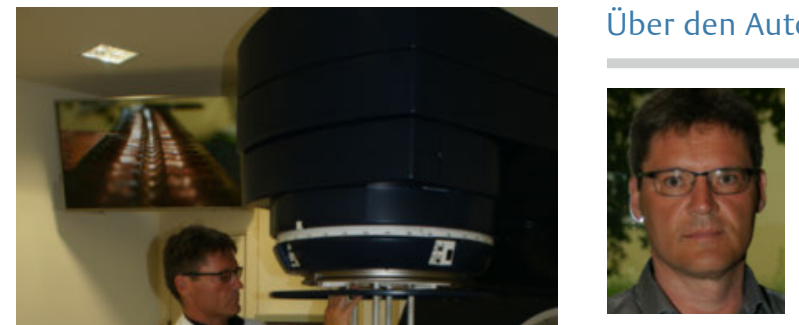

Hartmut Dörwaldt ist Fachlehrer für „Übungen Strahlentherapie" und „Übungen Bildverarbeitung“. Er absolvierte von 1991-1993 die Ausbildung MTRA am Klinikum Nürnberg. Von

1993-2004 arbeitete er im Diakoniekrankenhaus Schwäbisch Hall (Strahlentherapie), seit 2004 ist er an der Berufsfachschule für MTRA am Klinikum Nürnberg.

Korrespondenzadresse

\section{Hartmut Dörwaldt}

BFS für MTRA am Klinikum Nürnberg Prof.-Ernst-Nathan-Str. 1

90419 Nürnberg

Tel: 0911/398-3178

- Abb. 7 Hartmut Dörwaldt mit Patientenpuppe und Bestrahlungsgerät. Quelle: Katja Röhr.
E-Mail: hartmut.doerwaldt@klinikumnuernberg.de 